\title{
ВЫЧИСЛЕНИЕ КОЭФФИЦИЕНТА РАСПРОСТРАНЕНИЯ COVID-19 С ЭЛИМИНАЦИЕЙ НЕДЕЛЬНОГО ЦИКЛА
}

Кромер В.В.

\begin{abstract}
For the sake of excluding the weekly cycle when calculating the reproduction number of covid-19, it is proposed to calculate with a change in the formula recommended by Russian consumer protection agency Rospotrebnadzor, not according to the last 8 data, but according to the last 11 data, which increases the calculation accuracy by 7 times, the mean square error of the calculation changing from 0.072 to 0.010 .
\end{abstract}

Резюме. С целью исключения недельного цикла при вычислении коээфициента распространения covid-19 предлагается производить расчет с изменением рекомендованной Роспотребнадзором формулы, не по 8 последним данным, а по 11 последним данным, что повышает точность вычисления в 7 раз, средняя квадратичная погрешность вычисления изменяется с 0,072 до 0,010 .

В эпидемиологии заразность инфекционных заболеваний характеризуют безразмерным показателем - базовым репродуктивным числом, или индексом репродукции $R_{0}$. Понижение заразности заболевания вследствие иммунизации части населения отображается эффективным репродуктивным числом $R$.

Индекс репродукции вычисляется в зависимости от числа заражающих контактов в единицу времени $\beta$ и среднего инфекционного периода $\tau$.

$$
R_{0}=\beta \cdot \tau .
$$

Один из способов снижения распространения инфекции - уменьшение $\beta$ путем карантина, самоизоляции, комендантского часа, использования средств, затрудняющих передачу инфекционного начала (ношение медицинских масок, респираторов, защитных щитков, перчаток и т.п.), соблюдение социальной (санитарной) дистанции.

Роспотребнадзор рекомендует в ситуации пандемии нового коронавируса (covid-19), рассчитывать коэффициент репродукции (названный коэффициентом распространения $R$ - средним количество людей, инфицированных одним больные до его изоляции), путем деления суммы числа больных, зарегистрированных в субъекте за последние 4 суток, на сумму числа больных, зарегистрированных в субъекте за предыдущие 4 суток.

$$
R=\frac{X_{8}+X_{7}+X_{6}+X_{5}}{X_{1}+X_{2}+X_{3}+X_{4}},
$$

где $X_{1}-X_{8}-$ число зарегистрированных больных в субъекте за соответствующие сутки [1].

Из определения следует, что инфекционный период covid-19 принят равным 4 дням. 
При внешней правильности методики подсчета $R$, она обладает определенным недостатком, существенно ограничивающим валидность метода на предмет принятия административных решений по изменению режима ограничений/послаблений, поскольку значение $R$, в силу наличия социального недельного цикла поведения людей, а тем самым установления контактов и риска заражения, циклически колеблется по дням недели. Так, согласно датскому эндокринологу Хамбургеру, в недельном ритме меняется в организме уровень гормонов коры надпочечников - важнейших регуляторов многих процессов.

Наиболее значителен эффект недельного цикла заражения covid-19 в мегаполисах, поясним на примере Москвы.

На рисунке 1 представлен график числа ежедневно регистрируемых больных, начиная с 1 апреля 2020 года, вплоть до сегодняшнего дня, 18 июля (тонкая черная линия).

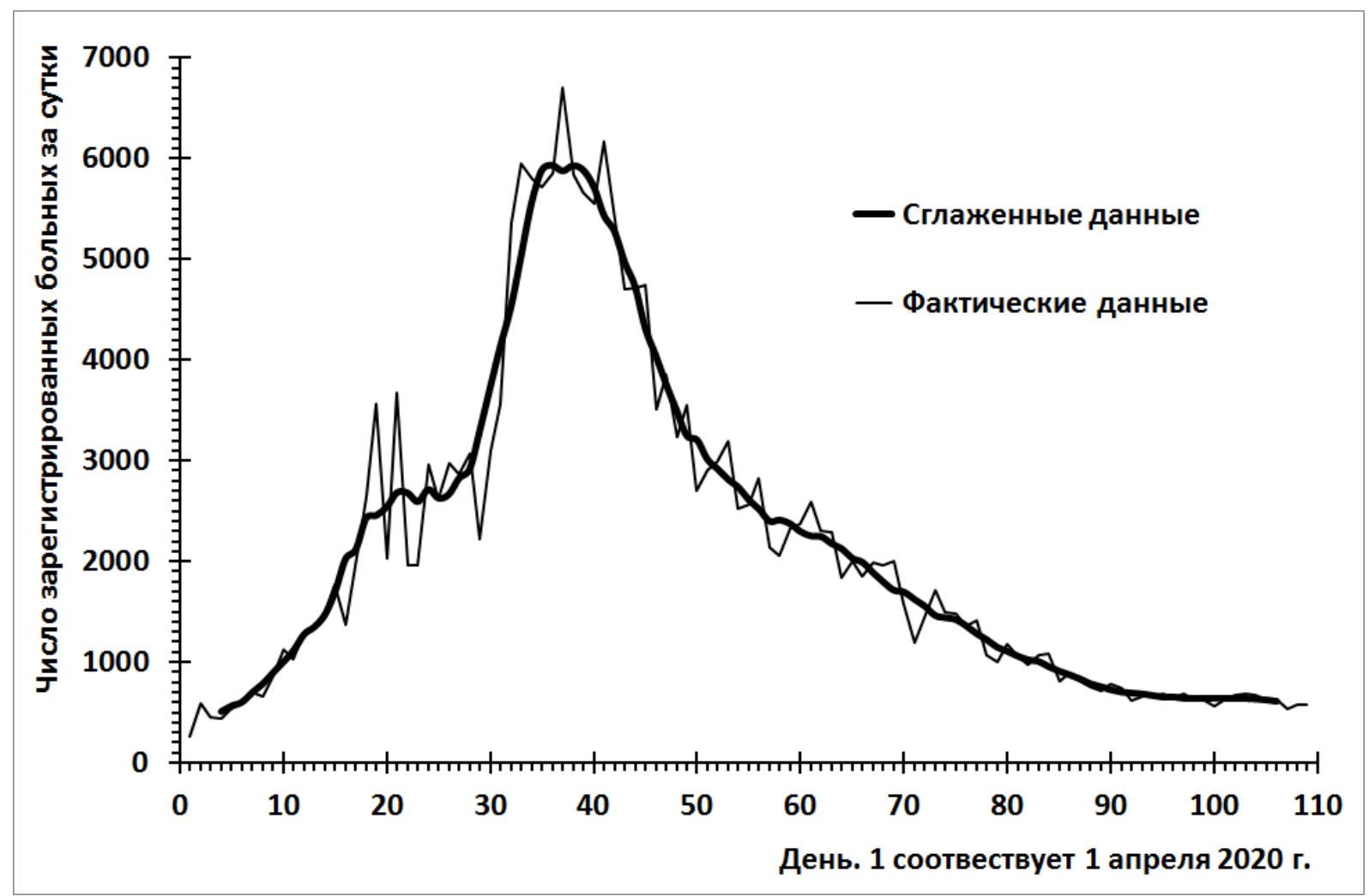

Рис. 1. Фактические и сглаженные данные числа зарегистрированных больных.

Выявляем, путем вычисления среднего арифметического с окном 7, вычищенный от недельного цикла тренд (толстая черная линия), и вычтем из фактических значений тренд (рисунок 2). 


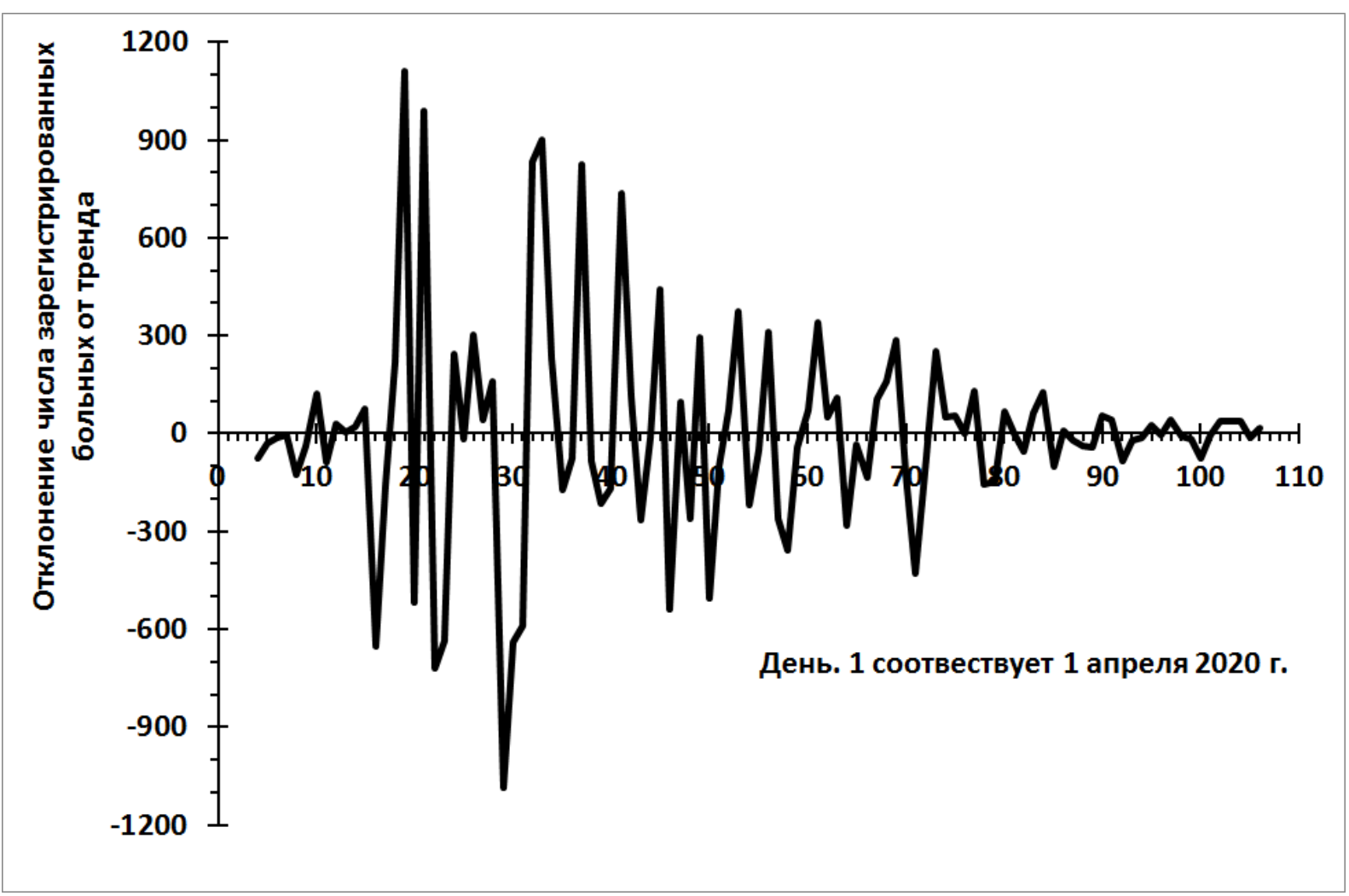

Рис. 2. Отклонение числа зарегистрированных больных от тренда по дням.

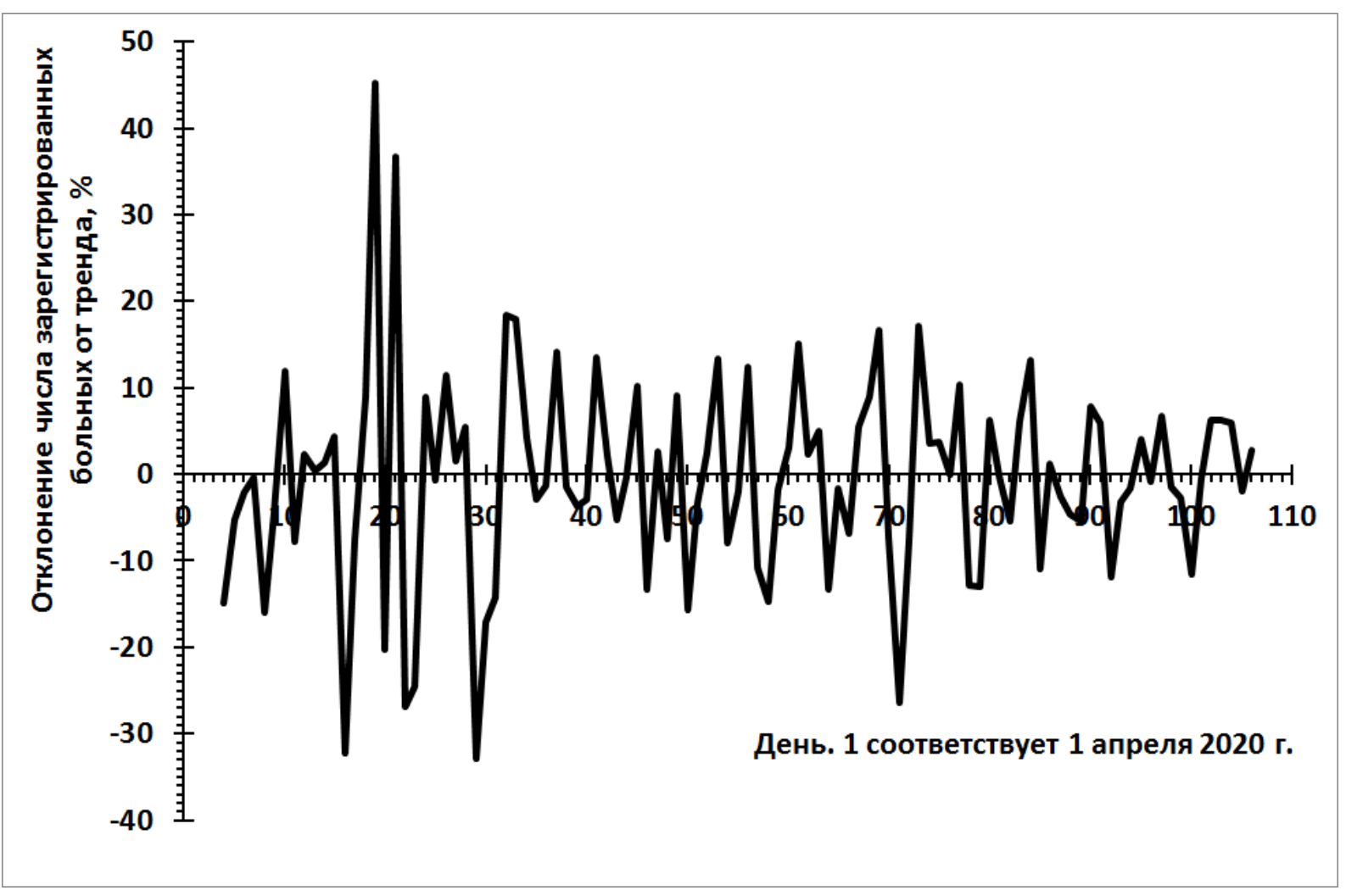

Рис. 3. Отклонение числа зарегистрированных больных от тренда по дням, в процентах. 
Налицо недельный цикл. Вычисляем относительные отклонение фактических значений от тренда по формуле

$$
\frac{X_{\phi}-X_{m}}{X_{m}} \cdot 100 \%,
$$

где $X_{\phi}$ - фактическое значение числа зарегистрированных больных, $X_{m}-$ значение по тренду. Результат в процентах отображен на графике (рисунок $3)$.

Находим среднее значение отклонения по дням недели (усреднение по 15 неделям), результат в виде гистограммы (рисунок 4). Отклонение достигает $7-12 \%$, в ту и другую сторону.

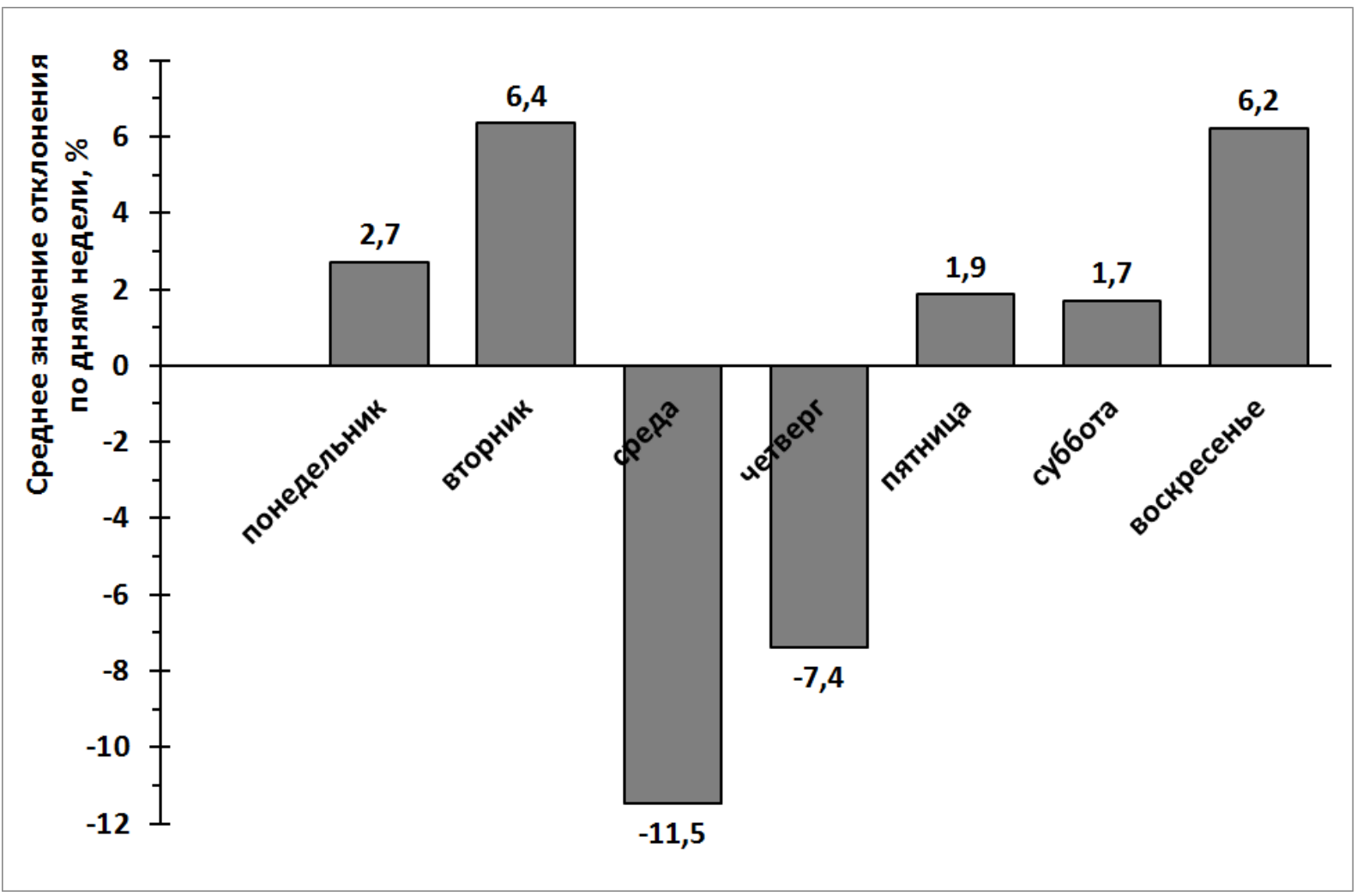

Рис. 4. Среднее значение отклонения числа зарегистрированных больных от тренда по дням недели, в процентах.

На графике (рисунок 5) рассчитан, по методике Роспотребнадзора, коэффициент распространения covid-19 по Москве (тонкая черная линия). Налицо наличие недельного цикла, что затрудняет принятие решения о распространении инфекции и требует откладывания решения, поскольку вычисленное в текущий день значение может существенно отклониться от тренда. 


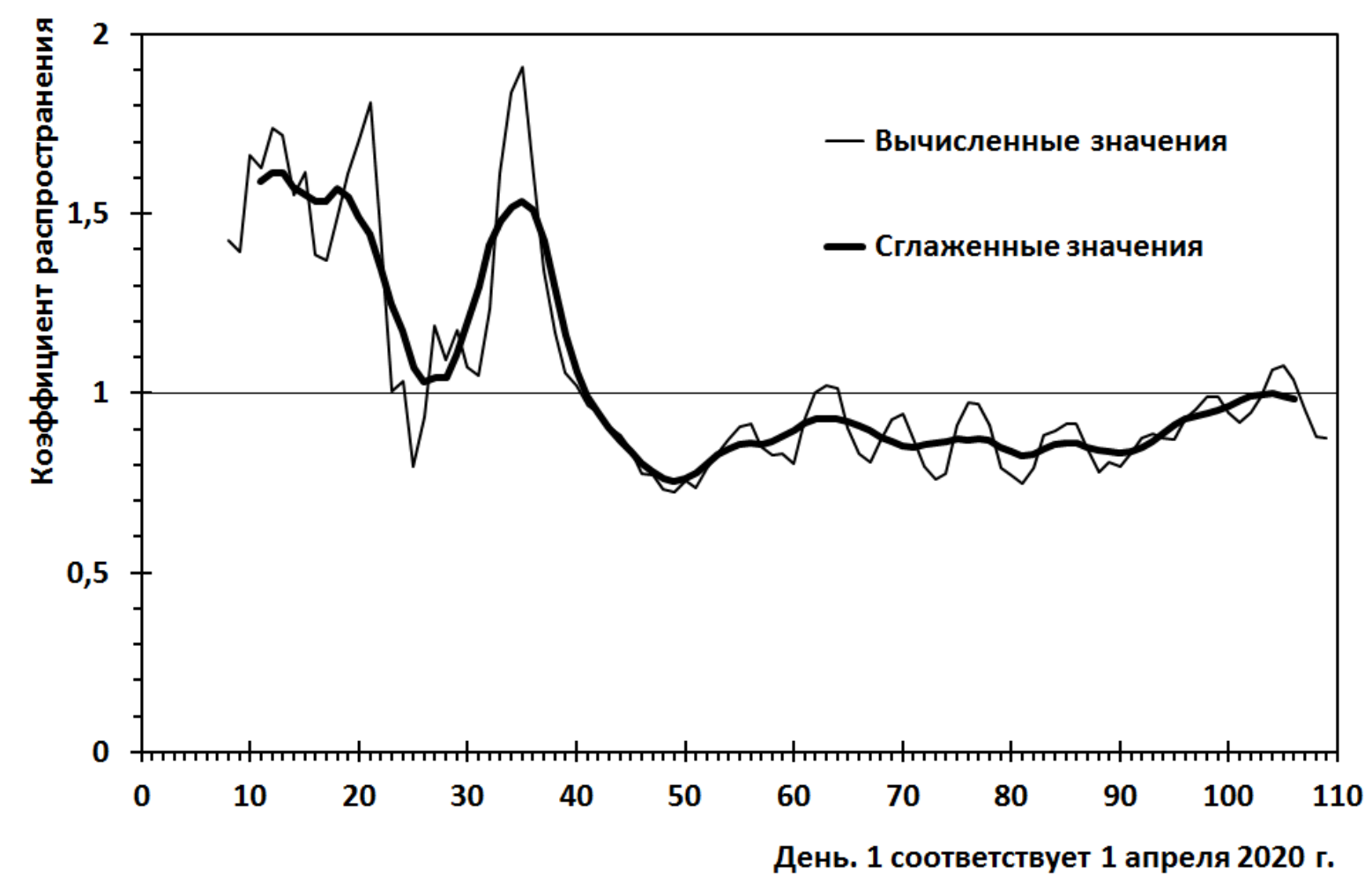

Рис. 5. Коэффициент распространения по дням.

Выясним величину этого отклонения, для чего найдем тренд (путем вычисления среднего арифметического с окном 7), толстая черная линия на графике рисунка 5, вычтем тренд из фактических значений и усредним по дням недели абсолютные отклонения вычисленных значений коэффициента распределения. Гистограмма на рисунке 6.

Налицо циклический характер изменения $R$ по дням недели, вполне объясняемый предыдущей гистограммой (рис. 4). Отклонения коэффициента распространения в сторону увеличения максимальны по вторникам, когда в числитель выражения (2) для $R$ попадают 4 значения чисел заболеваний за сутки (за вторник, понедельник, воскресенье, субботу), все ушедшие вверх от тренда, и в знаменателе сумма чисел заболеваний за пятницу, четверг, среду и вторник, с явным преобладанием (за счет четверга и пятницы) отклонений от тренда вниз.

Аналогично, отклонение коэффициента распространения в сторону уменьшения максимально в субботу (в числителе - значения за субботу, пятницу, четверг и среду с преимущественным отклонением вниз за счет среды и четверга), а в знаменателе - показатели за вторник, понедельник, воскресенье и субботу (все отклонения вверх). 


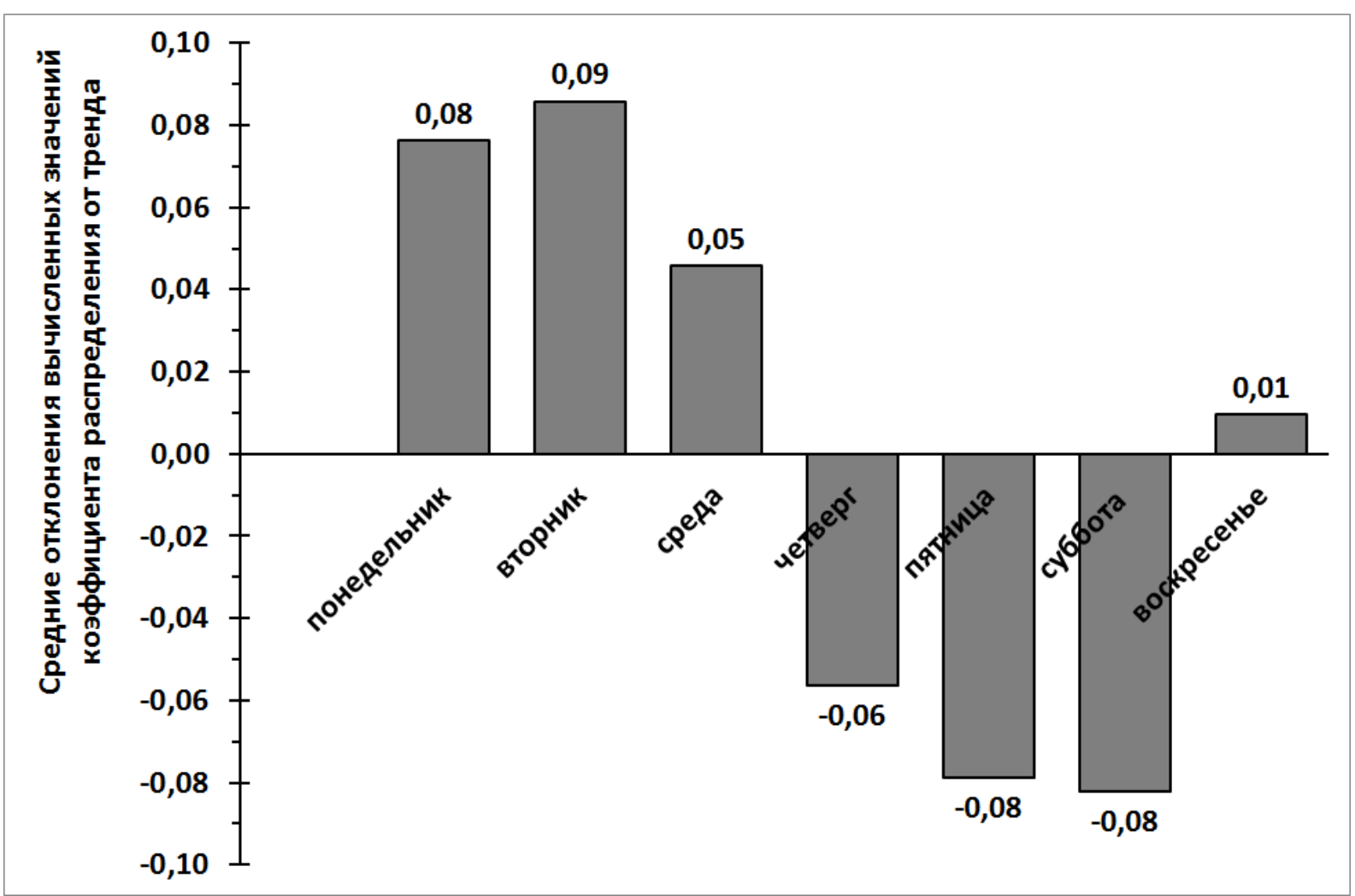

Рис. 6. Среднее отклонение вычисленных значений коэффициента распространения от тренда в зависимости от дня недели.

Отклонения коэффициента распространения вверх или вниз до $\pm 0,09$ неприемлемы для принятия решений, поскольку в случае наиболее критических показателей в окрестности $R=1$, решения при $R=1,09$ и $R=0,91$ заведомо принимаются разные.

По определению, недельный цикл элиминируется при подсчете коэффициента распространения для заболевания с инфекционным периодом, равным 7 дням. При инфекционном периоде в 4 дня цикличность может быть устранена вычислением коэффициента распространения $R$ по следующей формуле:

$$
R=\frac{X_{5}+X_{6}+X_{7}+X_{8}+X_{9}+X_{10}+X_{11}}{X_{1}+X_{2}+X_{3}+X_{4}+X_{5}+X_{6}+X_{7}}
$$

где $X_{1}-X_{11}$ - число зарегистрированных больных в субъекте за соответствующие сутки. В отличие от (2), где для вычисления $R$ требуется 8 последних значений, вычисления по (4) требуют 11 последних значений.

Валидность выражения (4) для $R$ поясняется тем, что если его представить как

$$
R=\frac{\left(X_{5}+X_{6}+X_{7}+X_{8}+X_{9}+X_{10}+X_{11}\right) / 7}{\left(X_{1}+X_{2}+X_{3}+X_{4}+X_{5}+X_{6}+X_{7}\right) / 7}
$$


то это отношение сглаженного с окном 7 значения 4-го по счету, начиная со дня вычисления, к 8-му по счету со дня вычисления.

Если вернуться к вычисляемому по (2) значению $R$, представленному в виде

$$
R=\frac{X_{8}+X_{7}+X_{6}+X_{5}}{X_{1}+X_{2}+X_{3}+X_{4}}=\frac{\left(X_{8}+X_{7}+X_{6}+X_{5}\right) / 4}{\left(X_{1}+X_{2}+X_{3}+X_{4}\right) / 4},
$$

то это отношение сглаженного с окном 4 значения, промежуточного между 2 и 3 по счету, начиная со дня вычисления, к промежуточному между 6 и 7 по счету со дня вычисления.

Таким образом, выражения по (4) и по (2) отличаются лишь тем, что выражение (4) запаздывает на 1,5 дня относительно выражения (2), что совершенно несущественно для целей регулирования [свойственное (2) запаздывание в 2,5 дня увеличивается до 4 дней при применении (4)].

При этом (4) имеет огромное преимущество для целей регулирования вследствие исключения недельного цикла. Для вычислений по формуле (4), как и по формуле (2), достаточно калькулятора на 4 действия, с незначительным увеличением количества действий по вводу данных.

Мы сознаем, что у формулы (4), в сравнении с формулой (2), ниже очевидная (лицевая, или фейс) валидность, что связано с вхождением значений $X_{5}, X_{6}, X_{7}$ одновременно и в числитель, и знаменатель выражения (4). Но это вхождение обосновано, и приводит к повышению конструктной валидности, т.е. формула оценивает именно то, что и должна оценивать - степень заразности инфекционного заболевания в данных условиях без зашумленности случайными колебаниями ввиду наличия недельного цикла.

На одном графике (рисунок 7) представлены вычисленные для каждого дня значения $R$ по двум сравниваемым методикам - традиционной (тонкая черная линия) по (2) и предлагаемой (толстая черная линия) по (4).

По графику видно, что при вычислениях по предлагаемой методике полностью элиминирован недельный цикл, значения коэффициента распространения по Москве устойчиво переходят 12 мая через значение $R=1$ сверху вниз, достигают 21 мая минимума $R=0,74$, а затем вновь возрастают к настоящему времени до значения $R=1,00$, что полностью отражает картину происходящего, со всеми ужесточениями и послаблениями режима самоизоляции, и позволяет более объективно принимать административные решения, связанные как с ограничением распространения инфекции, так и с необходимостью формирования коллективного иммунитета. 


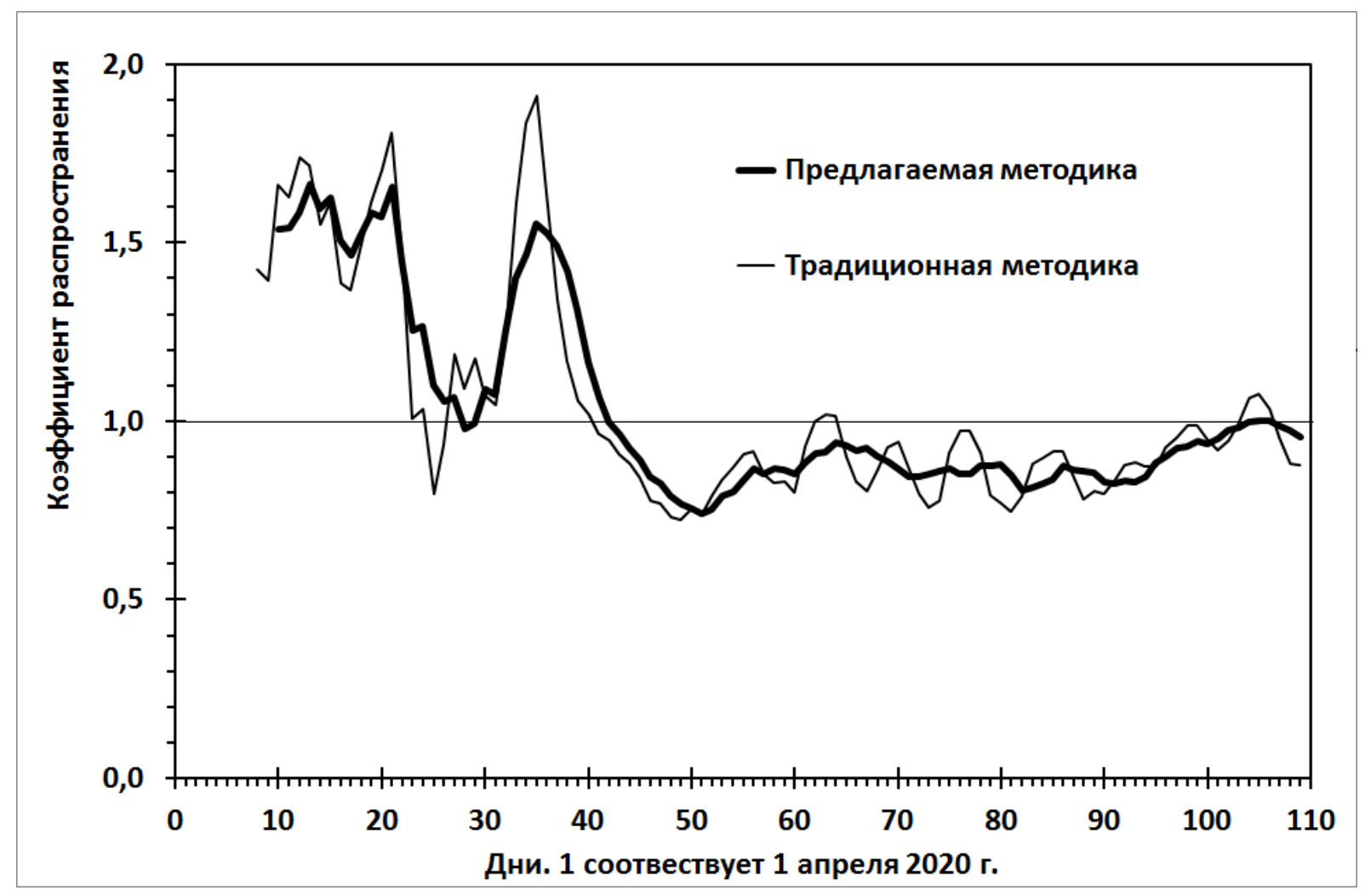

Рис. 7. Сравнение коэффициентов распространения, вычисляемых по традиционной и предлагаемой методикам.

Вычисленные традиционно значения порождают ложные иллюзии и ложные тревоги. Так, 2 июня значение $R$ якобы достигает 1,02, в то время как более правильное значение в окрестности этой даты не превосходит 0,94 ; наиболее показательны вычисленные традиционно значения 1,08 за 14 июля и 0,88 за 19 июля, что всего лишь артефакты, обусловленные недельным циклом - 14 июля вторник, а 19 июля суббота. Из рисунка 6 следует, что вторник - день максимума $R$, а суббота - минимума $R$, вычисляемых традиционно, с размахом 0,17. По факту, разница между двумя вычисленными традиционно отметками составила сопоставимую величину 0,20.

Два других аналогичных примера: вычисленный традиционно коэффициент распространения $R 5$ мая достигает значения 1,91, характерного для начального этапа развития covid-19 (исходя из значения базового репродуктивного числа $R_{0}=2$ ), в то время как фактически следует считаться с более безопасным значением 1,55.

Также, 25 апреля вычисленное традиционно значение 0,80 может побудить сделать вывод о существенном переломе тенденции, в то время как в окрестности этого минимума (28 апреля), реальное значение не опускается ниже 0,98 , да и то всего на 1 день, с превышением значения 1,00 в соседние дни. 
Характерно то, что среднее квадратичное отклонение вычисленных по традиционной методике значений $R$ составляет 0,072 , в то время как по предлагаемой методике 0,010 , что свидетельствует об уточнении вычисленных значений в 7,2 раза.

Цена вопроса по изменению методики окупается повышением валидности получаемых результатов для целей принятия административных решения и информирования населения, которое, в случае наступления следующих волн нового коронавируса либо социркуляции вирусов сезонного гриппа и коронавируса, будет только сбиваться с толку чередой ежедневно поступающих и противоречивых ввиду наличия случайной компоненты показателей.

Правильное информирование позволит на уровне обыденного сознания установить изменения коэффициента распространения с предыдущим поведением и соблюдением режима, с отставанием не более недели, что, несомненно, будет способствовать сознательному соблюдению рекомендаций Роспотребнадзора и властей ввиду установления в сознании высококоррелирующей взаимной связи.

Примечание: все расчеты и выводы основываются на официальных данных, поступающих в 10.00 мск времени на следующий за днем отчета день. Таким образом, если в тексте статьи и на гистограмме показано, что наибольшее число больных регистрируется во вторник и воскресенье, а наименьшее в среду и четверг, по факту речь идет о больных, поступивших в медицинские учреждения соответственно днем раньше.

\section{Список литературы}

1. Методические рекомендации для определения показателей, которые являются основанием для поэтапного снятия ограничительных мероприятий. https://www.rospotrebnadzor.ru/upload/MP_поэтапное снятие огранич._08.05.2020.pdf 